\title{
The Inclination Window for Low Earth Sun Synchronized Satellite Orbits
}

\author{
Shkelzen Cakaj, Bexhet Kamo ${ }^{a}$, Krešimir Malarićb
}

LEO (Low Earth Orbit) environmental satellites provide continuous coverage of Earth, supplying meteorological and oceanic observation data which are important in aerospace and maritime. The missions of such satellites are mainly based on photo imagery. For photo imagery, it is also important that the area observed from the satellite is treated under the same lighting conditions. This can be achieved by keeping the orbital plane position constant relative to the Sun due to the Earth's motion around the Sun, defined as orbital Sun synchronization. The line of nodes defines the orientation of the satellite's orbital plane in space. Nodal regression is defined as the shift of the orbit's line of nodes over time, as Earth revolves around the Sun. Nodal regression is caused by the Earth's oblateness. Nodal regression is a very useful feature, especially used to synchronize low Earth circular orbits with the Sun. Nodal regression depends on orbital attitude and orbital inclination angle. This paper provides an inclination window calculation for different attitudes in order to maintain orbital Sun synchronization.

\section{KEY WORDS}

$\sim$ LEO

$\sim$ Satellite

$\sim$ Inclination

$\sim$ Sun-synchronization

Faculty of Electrical and Computer Engineering, University of Prishtina, Kosovo e-mail: shkelzen.cakaj@fulbrightmail.org

a. Faculty of Information Technology, Polytechnic University of Tirana, Albania e-mail: bkamo@fti.edu.al

b. Faculty of Electrical Engineering and Computing, Zagreb University, Croatia e-mail: kresimir.malaric@fer.hr

\section{INTRODUCTION}

Generally, circular orbits of satellites are categorized as Geosynchronous Earth Orbits (GEO), Medium Earth Orbits (MEO) and Low Earth Orbits (LEO). The main difference between them is their attitude above the Earth's surface (Richharia, 1999; Roddy, 2006; Maral and Bousquet, 2002).

The Sun synchronization feature is typical for LEOs. Sun synchronized low Earth orbits have a very wide application in scientific missions based on photo imagery, like weather, Earth observation and space exploration (Oberright, 2004).

The specific application of LEO satellites is related to location determination in case of maritime distress. The satellite system is designed to raise distress alert and provide location data to assist in search and rescue operations. A satellite detects and locates distress beacons (406 MHz) activated at a distress location. The system calculats the location of a distress event using Doppler processing techniques (Cakaj et al., 2010).

A natural phenomenon like nodal regression effect enables orbital Sun synchronization (Chobotov, 2011). The satellite's position in space is defined by space orbital parameters, briefly described in the second section. The mathematical background of nodal regression is also provided. Finally, the last section deals with the defined conditions for sun synchronization to be attained at different orbital attitudes. The paper ends with the inclination window determination for sun synchronization of low Earth orbits.

\section{SPACE ORBITAL PARAMETERS}

The path of a satellite's motion is an orbit. In order to describe the satellite's movement within its orbit, the space orbital parameters need to be defined. These space orbital 


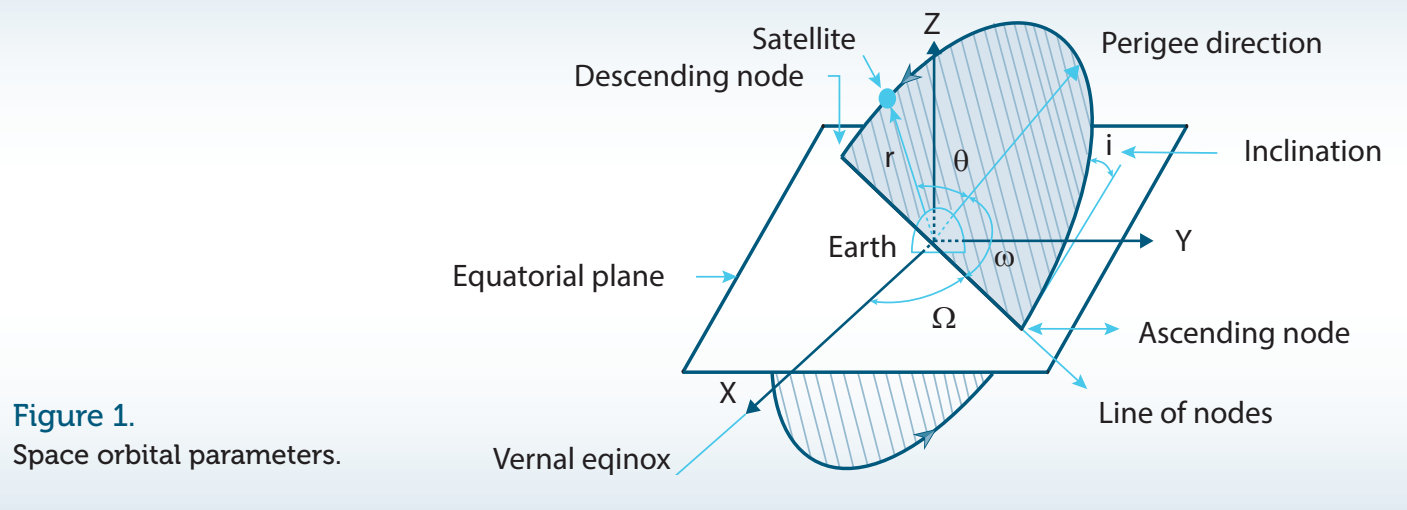

parameters are schematically presented in Figure 1 and defined under items a), b), c) and d) (Cakaj and Malaric, 2007; Cakaj et al., 2007).

\section{a) The position of the orbital plane in space.}

This is specified by means of two parameters - inclination $i$ and right ascension of the ascending node $\Omega$. Inclination $i$ represents the angle of the orbital plane with respect to the Earth's equator. The right ascension of the ascending node $\Omega$ defines the location of the ascending node. The ascending and descending orbital crossing nodes make a line of nodes, with respect to a fixed direction in space. The fixed direction is the Vernal equinox. The Vernal equinox is the direction of the line connecting the Earth's center and the Sun on the first day of spring (Richharia, 1999; Roddy, 2006; Maral and Bousquet, 2002).

b) Location of the orbit in orbital plane.

An infinite number of orbits can be laid within an orbital plane. Thus, the orientation of the orbit in its plane is defined by the argument of perigee $\omega$. This is the angle, taken positively from $0^{\circ}$ to $360^{\circ}$ in the direction of the satellite's motion, between the direction of the ascending node and the direction of the perigee (Richharia, 1999; Roddy, 2006; Maral and Bousquet, 2002).

c) Position of the satellite in orbit.

The position of the satellite in orbit is determined by the angle $\theta$ called the true anomaly, which is the angle measured positively in the direction of the satellite's movement from $0^{\circ}$ to $360^{\circ}$, between the direction of the perigee and the position of the satellite (Richharia, 1999; Roddy, 2006; Maral and Bousquet, 2002). d) The shape of orbit.

The shape of orbit is presented by the semi-major axis $a$ which defines the size of orbit and the eccentricity which defines the shape of the orbit.

\section{NODAL REGRESSION}

The Earth rotates towards the East to West. This is known as the eastward direction, while the opposite is called the westward direction. An orbit in which satellite moves in the same direction as the Earth's rotation is known as prograde or direct orbit. The inclination of a prograde orbit always lies between $0^{\circ}$ and $90^{\circ}$. Most satellites are launched in a prograde orbit because the Earth's rotational velocity provides part of the orbital velocity with the consequent saving of launch energy (Richharia, 1999; Roddy, 2006; Maral and Bousquet, 2002).

An orbit in which the satellite moves in direction opposite to that of the Earth's rotation is called retrograde orbit. The inclination of a retrograde orbit always lies between $90^{\circ}$ and $180^{\circ}$.

The Earth is not a spherical homogeneous body. The Earth is characterized by a bulge at the equator, and a slight flattening at the poles. The terrestrial potential at a point in space (in our case the point indicates a satellite) depends not only on the distance $r$ to the Earth's centre but also on the respective longitude and latitude. This terrestrial potential is expressed through geo-potential coefficients $J_{n}$, so called zonal harmonics. Zonal harmonics $J_{n}$ reflect the potential dependence on the latitude. The $J_{2}$ term due to the flattering of the Earth dominates all other terms. The values of these coefficients are given by different models. Based on GEM4 model it yields (Maral and Bousquet, 2002):

$J_{2}=1.0827 \cdot 10^{-3}$

The potential generated by the non-spherical Earth causes variations of the orbital elements. The most affected orbital elements are the argument of the perigee and the right ascension. Only the effect on the right ascension is considered in this paper. Under this effect, the right ascension of ascending node $\Omega$ is shifting. The line of nodes lying in the equatorial plane drifts about the center of the Earth; consequently the orbital plane shifts.

Nodal regression refers to the shift of the orbital plane over time, as the Earth revolves around the Sun. Nodal regression is a very useful feature especially utilized for circular orbits which it provides with Sun synchronization. An approximate expression for the nodal rate regression of $\Omega$ due to time is expressed as (Maral and Bousquet, 2002): 
$\frac{d \Omega}{d t}=-\left(\frac{3}{2}\right) n_{0} A J_{2} \cos i$

where:

$A=\frac{R_{E}^{2}}{a^{2}\left(1-e^{2}\right)^{2}}$

and $R_{E}=6378 \mathrm{~km}$ is Earth's radius, $e$ is orbital eccentricity, $i$ is the inclination, $a$ is a semi-major axis of a satellite's orbit and $n_{0}$ is mean movement of the satellite:

$n_{0}=\frac{2 \pi}{T}$

whith $T$ being orbital period. For circular orbits $e=0$ and $a=r$, where $r$ is the orbital radius of a circular orbit. Orbital period for circular orbits is expressed as:

$T=2 \pi \sqrt{\frac{r^{3}}{\mu}}$

where $\mu=3.986005 \cdot 10^{14} \mathrm{~m}^{3} / \mathrm{s}^{2}$ is the Earth's geocentric gravitational constant. For circular orbit yields:

$A=\frac{R_{E}^{2}}{r^{2}}$

Substituting (4), (5), and (6) at (2) and then considering values of $R_{E}, \mu$ and $J_{2}$, finally stems nodal regression expressed by inclination $i$ and orbital radius $r$. The nodal regression expressed in $\left[{ }^{\circ} /\right.$ day $]$ is (Basics of space flight, 2011; Tewari, 2007):

$\Delta \Omega=-2.06474 \cdot 10^{14} \cdot \frac{\cos i}{r^{7 / 2}}[\circ /$ day $]$

It follows from (7) that the nodal regression for circular orbits depends upon the orbit inclination and orbital altitude (radius). The nodal regression is zero if the inclination angle is $90^{\circ}$. Since when the orbit inclination angle is $i<90^{\circ}$, the deviation is negative, according to (2) the satellite orbital plane rotates in the direction opposite to the direction of the Earth's rotation. When the orbit inclination angle is $i>90^{\circ}$, the deviation is positive, so the satellite orbital plane rotates in the same direction as the direction of the Earth's rotation. Thus, if the orbit is prograde, the nodes slide westward, and if it is retrograde, the nodes slide eastward. This means that due to the above effect, the nodes (line of nodes) move in the direction opposite to the direction of satellite motion; hence the term nodal regression. When the rate of change is negative, the regression is westward and when the rate is positive the regression is eastward.

The nodal regression effect is typical for LEO orbits. For higher orbits this effect is negligible. The aim of this paper is to conclude the range of nodal regression for different LEO orbit attitudes under different inclination angles. Considering the Van Allen belt effect (above around $1400 \mathrm{~km}$ ) (Van Allen radiation
Table 1. Nodal regression [\%day].

\begin{tabular}{ccccc}
\hline Inclination & \multicolumn{4}{c}{ Orbital radius [km] } \\
\cline { 2 - 5 }$\left[{ }^{\circ}\right]$ & 7000 & 7200 & 7400 & 7600 \\
\hline 20 & -6.74 & -6.12 & -5.55 & -5.06 \\
\hline 30 & -6.21 & -5.64 & -5.12 & -4.66 \\
\hline 40 & -5.50 & -4.99 & -4.52 & -4.13 \\
\hline 50 & -4.61 & -4.19 & -3.78 & -3.46 \\
\hline 60 & -3.59 & -3.26 & -2.95 & -2.69 \\
\hline 70 & -2.45 & -2.22 & -2.02 & -1.84 \\
\hline 80 & -1.24 & -1.13 & -1.02 & -0.94 \\
\hline 90 & 0 & 0 & 0 & 0 \\
\hline 100 & 1.24 & 1.13 & 1.02 & 0.94 \\
\hline 110 & 2.45 & 2.22 & 2.02 & 1.84 \\
\hline 120 & 3.59 & 3.26 & 2.95 & 2.69 \\
\hline 130 & 4.61 & 4.19 & 3.78 & 3.46 \\
\hline 140 & 5.50 & 4.99 & 4.52 & 4.13 \\
\hline 150 & 6.21 & 5.64 & 5.12 & 4.66 \\
\hline 160 & 6.74 & 6.12 & 5.55 & 5.06
\end{tabular}

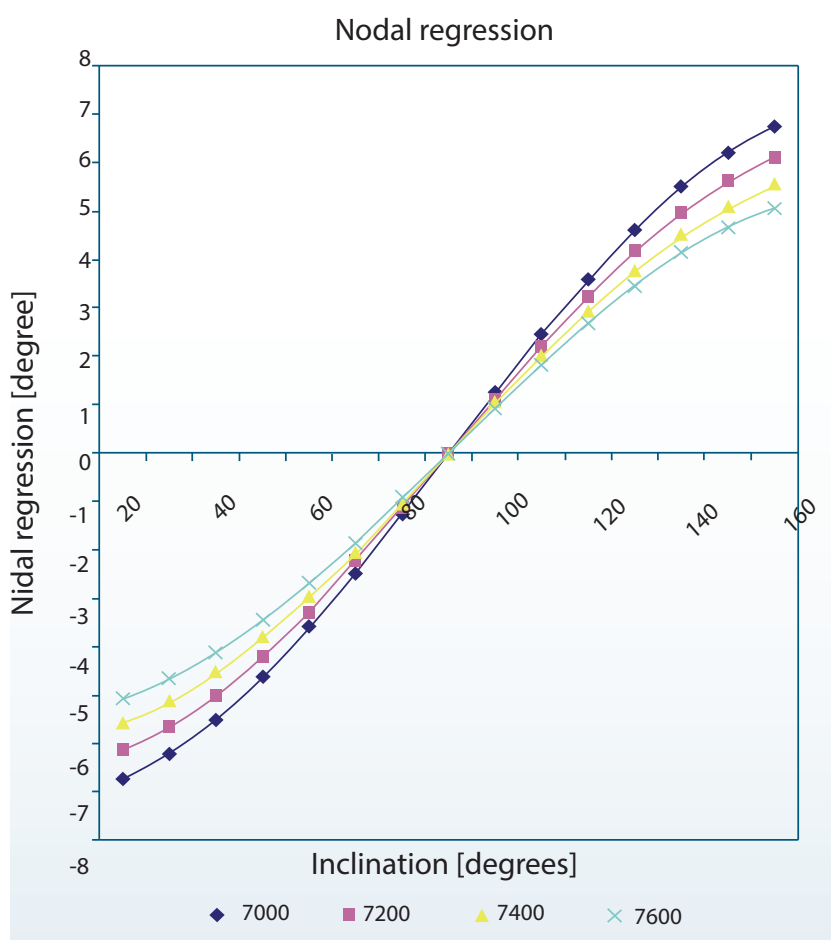

Figure 2. Nodal regression [\% day].

belts, 2012), attitudes from $600 \mathrm{~km}$ up to $1200 \mathrm{~km}$ are considered for simulation purposes. The eccentricity of LEOs is too low, and can be expressed as $e \approx 0$ and $a=r$ Thus, for attitudes between 
$600 \mathrm{~km}$ and $1200 \mathrm{~km}$, if the Earth's radius is rounded to $R_{E} \approx 6400$ $\mathrm{km}$, the orbits' radius range of between $7000 \mathrm{~km}$ and $7600 \mathrm{~km}$ is obtained. Considering (7), for such orbits' radius range the nodal regression [\%day] is calculated for different inclination angles $i$ and given in Table 1 and Figure 2.

The above results confirm that nodal regression for attitudes between $600 \mathrm{~km}$ and $1200 \mathrm{~km}$ may range from $0^{\circ}$ up to $6.7^{\circ}$ depending on the inclination angle. Lower inclination causes higher deviation. If inclination is $90^{\circ}$ there is no nodal deviation. Figure 2 illustrates that for eastward regression, inclination $i$ must be greater than $90^{\circ}$, consequently, the orbit must be retrograde. This enables the orbital plane to keep a constant angular relationship with the Sun throughout all the year's seasons. If the nodal regression causes a satellite's orbit to shift for about one degree per day throughout the year, the satellite's orbit will be aligned with the Sun. If this condition is met, the orbit is Sun synchronized.

\section{ORBITAL SUN SYNCHRONIZATION}

LEO satellites have a very wide application, e.g. for astronomical purposes, remote sensing of oceans, Earth's climate changes or Earth's imagery with high resolution (NOAA web page, 2012).

LEO environmental satellites are a part of the international Search and Rescue Satellite Aided Tracking (SARSAT) system. Ships and vessels must be equipped with an appropriate beacon which sends a signal to the satellite in case of distress. Each satellite pass transmits information about distress location. In cases of aircraft, marine or individual distress, the accuracy of location determination and the time required to alert rescue authorities depends on the reliability of communication between

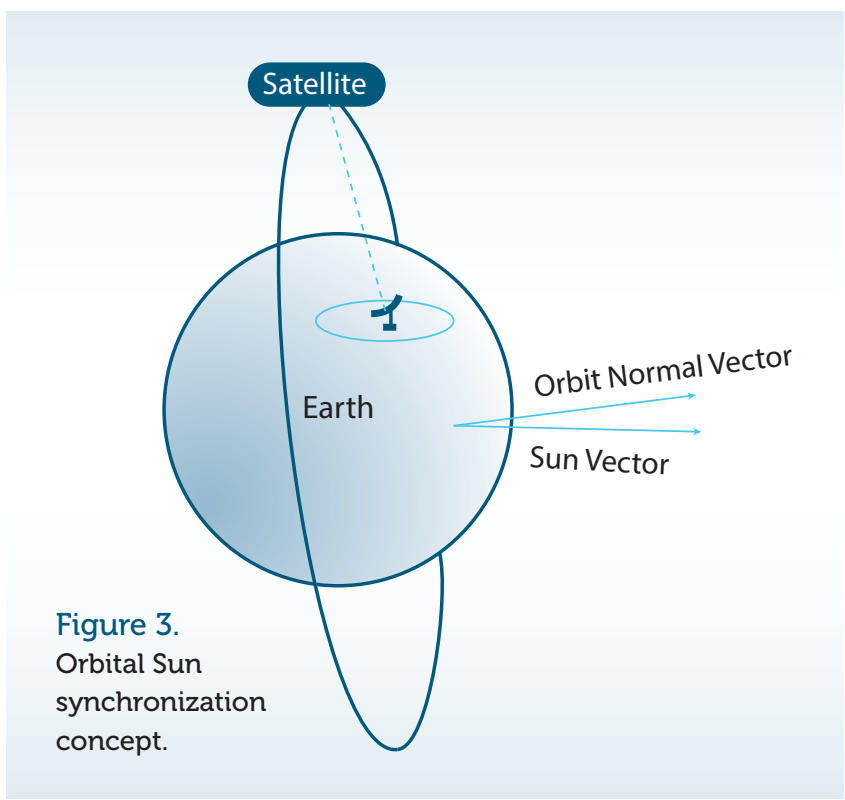

the ground terminals and satellites.

In order for the observed area to be treated under the same illumination conditions during satellite photo imagery missions, the position of the observed area relative to the Sun is very important. This could be achieved by keeping a constant Sun position related to the satellite's orbital plane. The angle between the normal orbital plane vector and normal Sun vector must be kept constant, as in Figure 3 (Zee and Stibrany, 2002).

A fixed orbital plane with respect to the Earth effectively makes a $360^{\circ}$ rotation in space in one year (about 365.25 days), since the Earth itself rotates around the Sun by $360^{\circ}$. This rate is equivalent to the rotation of the orbital plane of about 0.986 [\% day] (Zee and Stibrany, 2002). By choosing a pair of particular values of $i$ and $r$, an orbit the nodal regression of which varies each day by a quantity equal to the rotation of the Earth around the Sun may be obtained. Mathematically this is expressed as:

$\frac{d \Omega}{d t}=0.9856^{\circ} /$ day

$\Delta \Omega=-2.06474 \cdot 10^{14} \cdot \frac{\cos i}{r^{7 / 2}}=0.9856\left[{ }^{\circ} /\right.$ day $]$

The angle between the line of nodes of the orbits and the mean direction of the Sun obtained in this way remains constant throughout the year as presented in Figure 4 (Cakaj et al., 2009). Thus, the normal vector of the orbit and the vector of the Sun keep the same angularity during the year. The orbits which fulfill this condition are known as Sun synchronized orbits. This is a very useful application for observation of the Earth because the satellite always views a given location on the Earth under similar Sun illumination.

From Figure 2 it is obvious that (6) and (7) are fulfilled only for $i_{1} \succ 90^{\circ}$ respectively for retrograde orbits. By solving the (9) for

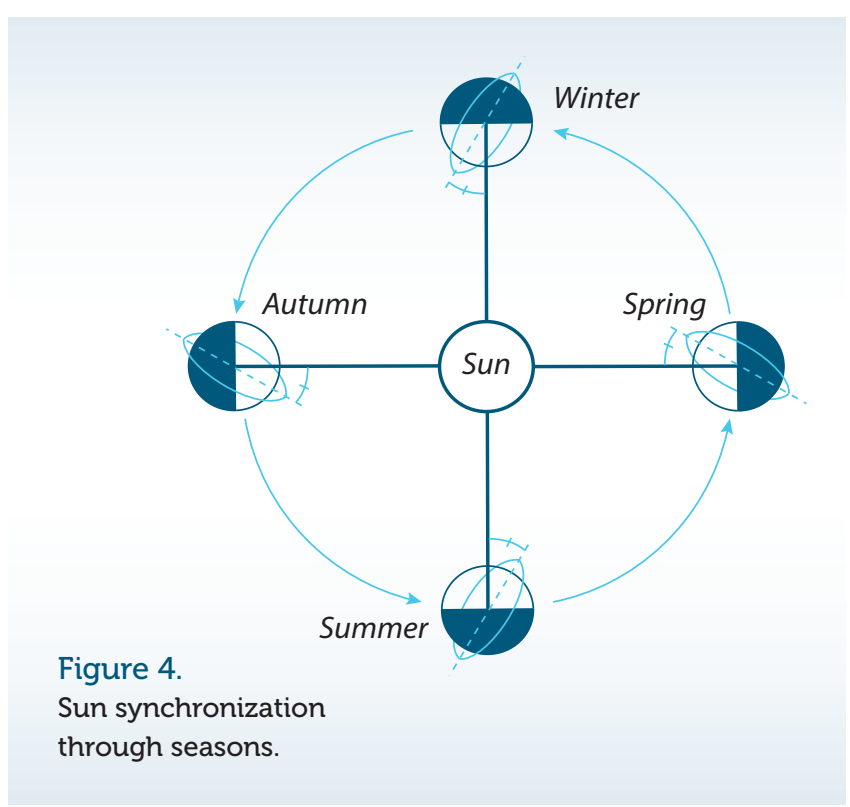


orbital attitude of $600 \mathrm{~km}$, consequently for $a=r=7000 \mathrm{~km}$ under no eccentricity $(e=0)$, the inclination for Sun synchronization will be:

$i_{1}=97.9^{\circ}$

and for orbital attitude of $1200 \mathrm{~km}$, consequently for $a=r=7000 \mathrm{~km}$ under no eccentricity $(e=0)$ the inclination for Sun synchronization will be:

$i_{2}=100.5^{\circ}$

Table 2. Nodal regression [ $\%$ day].

\begin{tabular}{ccccc}
\hline $\begin{array}{c}\text { Inclination } \\
{\left[{ }^{\circ}\right]}\end{array}$ & 7000 & 7200 & 7400 & 7600 \\
\hline 97 & 0.876 & 0.793 & 0.721 & 0.656 \\
\hline 98 & 1.001 & 0.906 & 0.824 & 0.750 \\
\hline 99 & 1.125 & 1.018 & 0.926 & 0.843 \\
\hline 100 & 1.248 & 1.131 & 1.028 & 0.936 \\
\hline 101 & 1.372 & 1.242 & 1.129 & 1.028 \\
\hline
\end{tabular}

Considering these values of inclination, as well as the range of lower and higher orbital attitudes, the nodal regression for the inclination range between $97^{\circ}$ and $101^{\circ}$ is presented in Table 2 and Figure 5. From Figure 5, for nodal regression of $0.986^{\circ} /$ day for LEO attitudes of between $600 \mathrm{~km}$ and $1200 \mathrm{~km}$ (radius between $7000 \mathrm{~km}$ and $7600 \mathrm{~km}$ ), the inclination range between $97.9^{\circ}$ and $100.5^{\circ}$ is obtained, and consequently the inclination window having the width of $2.6^{\circ}$.

\section{CONCLUSIONS}

Nodal regression is a very useful feature, especially utilized to synchronize circular orbits with the Sun. Sun synchronized orbits are used for satellites the missions of which are mainly based on photo imagery, also having application in maritime.

Sun synchronization feature is typical for LEOs. At medium and high orbits (MEO or GEO) the nodal regression effect is negligible, and such orbits cannot be Sun synchronized.

Sun-synchronization for circular orbits depends on attitude and inclination and is retrograde. The orbital Sun synchronization is achieved through inclination angles ranging between $97.9^{\circ}$ and $100.5^{\circ}$ for LEO attitudes between $600 \mathrm{~km}$ and $1200 \mathrm{~km}$. The inclination window for sun synchronization has the width of $2.6^{\circ}$ for attitudes ranging between $600 \mathrm{~km}$ and $1200 \mathrm{~km}$.

\section{REFERENCES}

Richharia, M., (1999), Satellite communication systems, New York: McGraw Hill.

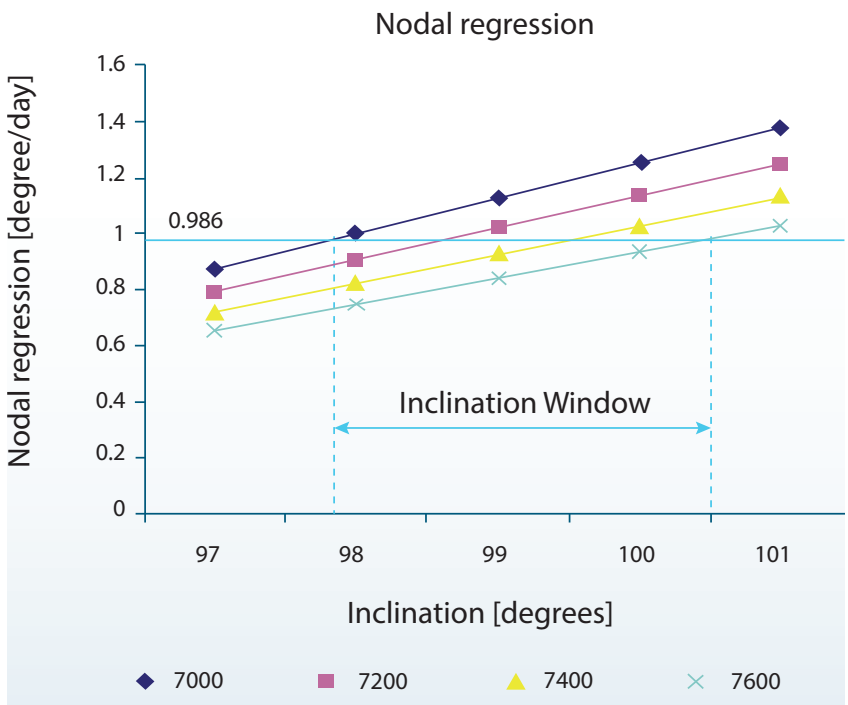

Figure 5. Inclination window.

Maral, G. and Bousquet, M., (2002), Satellite communication systems, Chichester: John Willey \& Sons

Oberright, J.E., (2004), Satellite artificial,World Book Online Reference Center, available at: http://mynasa.nasa.gov/worldbook/artificial_satellites_worldbook. html, [accessed 12 September 2012.].

Cakaj, S., Fitzmaurice, M., Reich, J. and Foster, E., (2010), Simulation of Local User Terminal Implementation for Low Earth Orbiting (LEO) Search and Rescue Satellites, The Second International Conference on Advances in Satellite and Space Communications SPACOMM 2010, Athens, June 13-19, pp. 140-145.

Chobotov, V. A., (2011), Orbital Mechanics, 3rd ed., Reston: American Institute of Aeronautics and Astronautics.

Cakaj, S. and Malaric, K., (2007), Rigorous analysis on performance of LEO satellite ground station in urban environment, International Journal of Satellite Communications and Networking, 25(6), pp. 619-643. http://dx.doi.org/10.1002/sat.895

Cakaj, S., Keim, W. and Malaric, K., (2007), Communication duration with low Earth orbiting satellites, Proc. IEEE, IASTED 4th International on Antennas, Radar and Wave Propagation, Montreal, Canada, May 30 - June 1, pp. 85-88.

Basics of space flight: Orbital mechanics, pp. 14, available at: http://www.braeunig. us/space/orbmech.htm, [accessed 12.04. 2011.].

Tewari, A., (2007), Atmospheric and space flight dynamics: Modelling and Simulation with Matlab and Simulink, Boston: Birkhäuser.

Van Allen radiation belts, available at: http://www.answers.com/topic/van-allenradiation-belt, [accessed 14 November 2012.].

NOAA web page, available at: http://www.noaa.gov, [accessed 14. 11. 2012.].

Zee, R. E., Stibrany, P., (2002), Canada's First Microsatellite - An Enabling LowCost Technology for Future Space Science and Technology Missions, Canadian Aeronautics and Space Journal, 48(1), pp. 1-11.,

http://dx.doi.org/ 10.5589/q02-008

Cakaj, S., Fischer, M., and Schotlz, A. L., (2009), Sun synchronization of Low Earth Orbits (LEO) through inclination angle, Proc. 28th IASTED International Conference on Modelling, Identification and Control, MIC 2009, Innsbruck, Austria, February 16 - 18, pp. 155-161. 